\title{
Tripartite Left Bundle Branch Area Pacing
}

\author{
Eriko Hasumi, ${ }^{1} \mathrm{MD}$ and Katsuhito Fujiu, ${ }^{1} \mathrm{MD}$
}

(Int Heart J 2021; 62: 1-3)

$\mathrm{P}$ ermanent His bundle pacing (HBP) brings us the most physiologic contraction in ventricular pacing. It reduces the risk for pacing-induced cardiomyopathy, heart failure hospitalization, and mortality compared to RV apical or septal pacing. ${ }^{1)}$ The AHA/ACC/HRS guideline on management of bradycardia recommends HBP as a class IIa indication for patients with atrioventricular (AV) block whose left ventricular (LV) ejection fraction is between $36 \%$ and $50 \%$. The guideline also considers HBP as a class IIb indication in patients with $\mathrm{AV}$ block at the $\mathrm{AV}$ node level who require ventricular pacing. ${ }^{2)}$

While additional potential benefits of HBP such as applications even for infra-nodal block, correction of left bundle branch block, ${ }^{3)}$ and alternative treatment to biventricular pacing, HBP may have some technical concerns with respect to being a routine clinical strategy. For example, the higher the capture threshold at implant, the greater the risk for a late rise in HBP thresholds, which need lead revisions. ${ }^{1)}$

The success rates of HBP lead implantation by trainees are limited due to a long learning curve, higher capture thresholds at implant, and increased risk for late lead trouble. The implantation technique of HBP requires outstanding expertise because target sites are a small area. Moreover, in a dilated heart, the target lesion access becomes challenging with the current delivery system, resulting in long procedural and fluoroscopic duration. The HBP success rate varies from $56 \%$ to $95 \%{ }^{4}$. It depends on the severity of conduction system impairment and the experience of the center. ${ }^{4)}$ Thus, some of the patients might have resulted in the loss of His capture during follow-up.

To overcome these issues, Huang, et al. first demonstrated a new strategy to directly capture the left ventricle conduction system called left bundle branch area pacing (LBBAP) ${ }^{5)}$ The LBBAP is achieved by deeply inserting a pacing lead from the right ventricular basal septum to the left side. The newly emerging pacing features are 1) LBBAP can contract the left ventricle through the conduction system 2) the target area is much broader than that of HBP, and 3) the pacing threshold is lower than that of $\mathrm{HBP}^{5)}$
Left bundle branch block correction is a benefit of HBP compared to traditional pacing. LBBAP has a further advantage for the correction because the left bundle beyond the blocked site can often be stimulated by placing the lead at a more distal location within the conduction system. ${ }^{5}$ Currently, LBBAP might be an alternative method for failed HBP cases such as high pacing threshold of the His bundle, infranodal atrioventricular block to show the exit block, or failed correction of left bundle branch block by HBP.

Huang, et al. proposed success criteria for LBBAP. ${ }^{6}$ Successful LBBAP could be confirmed when the following criteria 1 and 2, and at least 1 of the other 3 criteria are met: 1) paced morphology of right bundle branch block pattern, 2) presence of left bundle branch potential, 3) left ventricular activation time (LVAT), defined as the interval from the pacing stimulus to the $\mathrm{R}$ wave peak in $\mathrm{V}$ $5 / 6$, is shortened at high output pacing, 4) availability of determination of selective and non-selective left bundle branch capture, and 5) evidence for direct left bundle branch capture. ${ }^{\text {) }}$

In selective left bundle branch pacing, pacing latency: stimulus-QRS complex, and discrete local endocardial activation wave separated from stimulus can be seen. This indicates capturing only the left bundle branch as a direct left bundle branch capture. In non-selective left bundle branch pacing, no stimulus-QRS latency is detected due to the result of capturing both the left bundle branch and the adjacent local left ventricular septal myocardium.

\section{Article p.78}

Zhang, et al. advocate that LBBAP could be classified into 3 classifications; left bundle branch pacing (LBBP), incomplete LBBP (icLBBP), or deep septal pacing (DSP) (Figure). ${ }^{7)}$ According to morphological changes of paced QRS while decrementing pacing output, they define these types as the following; 1) LBBP, capturing only the left bundle branch at low output pacing, is equivalent to selective left bundle branch pacing as defined by the past reports. 2) icLBBP can capture both the left bundle branch and the adjacent local septal myocardium at high output pacing but show left bundle branch capture loss at

From the ${ }^{1}$ Department of Cardiovascular Medicine, Graduate School of Medicine, The University of Tokyo, Tokyo, Japan.

Address for correspondence: Eriko Hasumi, MD or Katsuhito Fujiu, MD, Department of Cardiovascular Medicine, Graduate School of Medicine, The University of Tokyo, 7-3-1 Hongo, Bunkyo-ku, Tokyo 113-8655, Japan. E-mail: ehasumi-circ@umin.ac.jp or fujiu-tky@umin.ac.jp

Received for publication December 4, 2020. Revised and accepted December 4, 2020.

doi: $10.1536 /$ ihj.20-771

All rights reserved by the International Heart Journal Association. 
A

Deep Septal Pacing

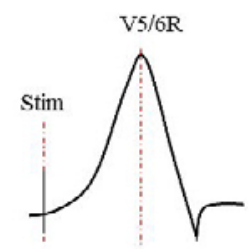

Long Stim-V5/6R

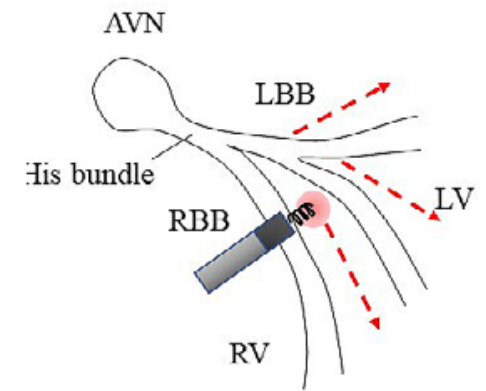

B

Incomplete LBBP

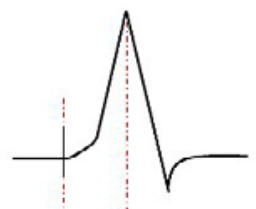

Short Stim-V5/6R without latency

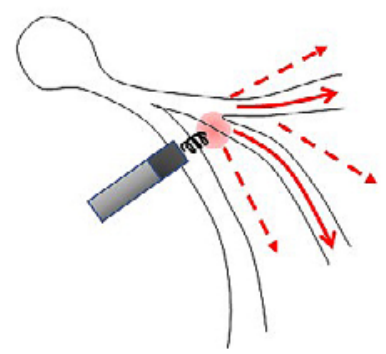

C Selective LBBP

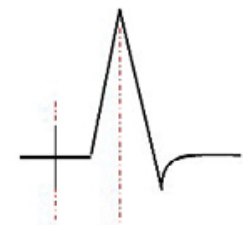

Short Stim-V5/6R with latency

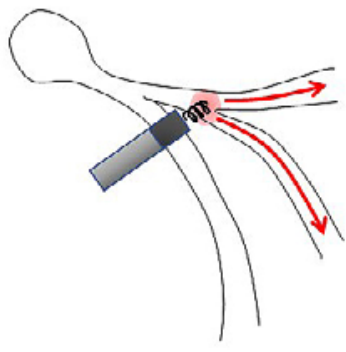

Figure. Paced QRS morphological features with the left bundle branch area pacing (LBBAP). A: Deep septal pacing (DSP) shows long Stim-V5/6R with a slur. DSP only captures LV (red area). B: Incomplete LBBP, short Stim-V5/6R with a slur. The tip of the lead is in the LV septum near the left bundle branch. Low output only captures LV. High output simultaneously captures LV and left bundle branch (red area). C: LBBP, short Stim-V5/6R with latency but not a slur. The lead's tip is near the left bundle branch. The low output shows selective left bundle branch pacing: LBBP (capture area: red area). The high output should show non-selective LBBP.

low output pacing. 3) DSP captures LV septal myocardium without left bundle branch capture regardless of pacing output. In this classification, DSP has been defined from RV septal pacing by a relative narrow paced QRS with RBBB morphology. Their overall success rate of LBBAP implantation was $95.5 \%$. The patients in whom LBBAP was attempted resulted in $20.4 \%$ with LBBP, $55.3 \%$ with icLBBP, and $24.3 \%$ with DSP. Although they did not address cardiac function and long-term efficacy, these 3 pacing modalities have no differences in mid-term parameters, including pacing threshold and safety. Both LBBP and icLBBP can capture the left bundle branch. However, the primary differential points might be whether the lead's tip is placed on the left bundle branch or left septum near the left bundle branch.

One of the recent discussions on LBBAP is whether the detection of left bundle potential during LBBAP is needed for successful LBBAP or not. The left bundle branch potential was only observed in $26.7-80 \%$ of patients with LBBP achieved. ${ }^{89}$ Other reports also demonstrated that left bundle branch potential was not necessary for successful LBBAP, especially in non-selective LBBP. ${ }^{10)}$ Considering these cases, the achievement of non-selective left bundle branch pacing may become more manageable than the procedure according to the current success criteria.

LBBAP could also be an alternative to CRT, at least in some cases. A few studies have showed that LBBAP corrected the left ventricular dyssynchrony in patients with left bundle branch block. ${ }^{10,11)}$ However, in the point of the left ventricular synchronization, there are still several veiled insights into LBBAP. The differences in the ef- fect on LV contraction and long-term prognosis between non-selective and selective LBBP remains unclear.

The effect of DSP on cardiac performance is another interesting discussion. Mafi-Rad, et al. reported the QRS duration by DSP is narrower than RV septal pacing. ${ }^{12)}$ Of course, paced QRS by DSP was slightly wider than that of LBBP. However, the prognostic differences between DSP and LBBP may have to be explored. If the LV synchrony under LBBAP and the long-term benefit of LBBAP were proved, it would surely be an alternative for failed HBP cases or a portion of CRT patients. In addition, the advantage of DSP over LBBP may also have to be analyzed.

Further investigation for mechanistic insight and clinical outcomes of LBBAP is warranted. By revealing these aspects, LBBAP would be a more common procedure to achieve physiological pacing of the LV.

\section{Disclosure}

Conflicts of interest: None.

\section{References}

1. Vijayaraman P, Naperkowski A, Subzposh FA, et al. Permanent His-bundle pacing: Long-term lead performance and clinical outcomes. Heart Rhythm 2018; 15: 696-702.

2. Kusumoto FM, Schoenfeld MH, Barrett C, et al. 2018 ACC/ AHA/HRS Guideline on the Evaluation and Management of Patients With Bradycardia and Cardiac Conduction Delay: Executive Summary: A Report of the American College of Cardiology/American Heart Association Task Force on Clinical Practice 
Guidelines, and the Heart Rhythm Society. J Am Coll Cardiol 2019; 74: 932-87.

3. Sharma PS, Dandamudi G, Herweg B, et al. Permanent Hisbundle pacing as an alternative to biventricular pacing for cardiac resynchronization therapy: A multicenter experience. Heart Rhythm 2018; 15: 413-20.

4. Sharma PS, Vijayaraman P, Ellenbogen KA. Permanent His bundle pacing: shaping the future of physiological ventricular pacing. Nat Rev Cardiol 2020; 17: 22-36.

5. Huang W, Su L, Wu S, et al. A Novel Pacing Strategy With Low and Stable Output: Pacing the Left Bundle Branch Immediately Beyond the Conduction Block. Can J Cardiol 2017; 33: 1736.e1-3.

6. Huang W, Chen X, Su L, Wu S, Xia X, Vijayaraman P. A beginner's guide to permanent left bundle branch pacing. Heart Rhythm 2019; 16: 1791-6.

7. Zhang J, Sheng X, Pan Y, Wang M, Guosheng Fu. Electrophysiological Insights into Three Modalities of Left Bundle Branch Area Pacing in Patients Indicated for Pacing Therapy. Int Heart
J 2021; 62: 78-86.

8. Cai B, Huang X, Li L, et al. Evaluation of cardiac synchrony in left bundle branch pacing: Insights from echocardiographic research. J Cardiovasc Electrophysiol 2020; 31: 560-9.

9. Li X, Li H, Ma W, et al. Permanent left bundle branch area pacing for atrioventricular block: Feasibility, safety, and acute effect. Heart Rhythm 2019; 16: 1766-73.

10. Hasumi E, Fujiu K, Nakanishi K, Komuro I. Impacts of Left Bundle/Peri-Left Bundle Pacing on Left Ventricular Contraction. Circ J 2019; 83: 1965-7.

11. Zhang W, Huang J, Qi Y, et al. Cardiac resynchronization therapy by left bundle branch area pacing in patients with heart failure and left bundle branch block. Heart Rhythm 2019; 16: 1783-90.

12. Mafi-Rad M, Luermans JG, Blaauw Y, et al. Feasibility and Acute Hemodynamic Effect of Left Ventricular Septal Pacing by Transvenous Approach Through the Interventricular Septum. Circ Arrhythm Electrophysiol 2016; 9: e003344. 